Article

\title{
Constructions of Helicoidal Surfaces in Euclidean Space with Density
}

\author{
Dae Won Yoon ${ }^{1}$, Dong-Soo Kim ${ }^{2}$, Young Ho Kim ${ }^{3}$ and Jae Won Lee ${ }^{1, *}$ \\ 1 Department of Mathematics Education and RINS, Gyeongsang National University, Jinju 52828, Korea; \\ dwyoon@gnu.ac.kr \\ 2 Department of Mathematics, Chonnam National University, Gwangju 61186, Korea; dosokim@chonnam.ac.kr \\ 3 Department of Mathematics, Kyungpook National University, Daegu 41566, Korea; yhkim@knu.ac.kr \\ * Correspondence: leejaew@gnu.ac.kr; Tel.: +82-55-772-2251
}

Received: 20 July 2017; Accepted: 18 August 2017; Published: 28 August 2017

\begin{abstract}
Our principal goal is to study the prescribed curvature problem in a manifold with density. In particular, we consider the Euclidean 3-space $\mathbb{R}^{3}$ with a positive density function $e^{\phi}$, where $\phi=-x^{2}-y^{2},(x, y, z) \in \mathbb{R}^{3}$ and construct all the helicoidal surfaces in the space by solving the second-order non-linear ordinary differential equation with the weighted Gaussian curvature and the mean curvature functions. As a result, we give a classification of weighted minimal helicoidal surfaces as well as examples of helicoidal surfaces with some weighted Gaussian curvature and mean curvature functions in the space.
\end{abstract}

Keywords: manifold with density; weighted curvature; helicoidal surface

\section{Introduction}

Differential geometers have been of interest in studying surfaces of constant mean curvature and constant Gaussian curvature in space forms for a long time. As a generalization of surfaces with constant Gaussian curvature or mean curvature, Kenmotsu [1], who generalized an old result of Delaunay [2], constructed surfaces of revolution with the mean curvature as a smooth function. A helicoidal surface in the Euclidean 3-space $\mathbb{R}^{3}$ is defined as the orbit of a plane curve under a helicoidal motion. As for helicoidal surfaces in $\mathbb{R}^{3}$, the surfaces with prescribed mean or Gaussian curvature have been studied by Baikoussis and Koufogiorgos [3]. On the other hand, Beneki et al. [4] and Ji and Hou [5-7] extended it in a Minkowski space. Recently, in [8], Yoon et al. also constructed helicoidal surfaces in a Heisenberg group for such a case.

A density on a Riemannian manifold is a positive function $\Phi$, weighting both volume and surface area. In terms of the underlying Riemannian volume $d V_{0}$ and area $d A_{0}$, the weighted volume and area are given by $d V=\Phi d V_{0}$ and $d A=\Phi d A_{0}$, respectively. Manifolds with densities (called also a weighted manifold) arise naturally in geometry as quotients of other Riemannian manifolds, in physics as spaces with different media, in probability as the famous Gauss space $\mathbb{G}^{3}$ with $\Phi=c e^{a^{2} r^{2}}$ for $a, c \in \mathbb{R}$ and $r^{2}=x^{2}+y^{2}+z^{2}$. Also, it was instrumental in Perelman's proof of the Poincare conjecture [9].

By using the first variation of the weighted area, the mean curvature $H_{\phi}$ of a surface in the Euclidean 3-space $\mathbb{R}^{3}$ with density $\Phi=e^{\phi}$ can be defined. It is given by

$$
H_{\phi}=H-\frac{1}{2}\langle\mathbf{N}, \nabla \phi\rangle,
$$

where $H$ and $\mathbf{N}$ are the mean curvature and the unit normal vector of a surface and $\nabla \phi$ is the gradient of $\phi$, which is called the weighted mean curvature or the $\phi$-mean curvature of a surface. The weighted mean curvature $H_{\phi}$ of a surface in $\mathbb{R}^{3}$ with density $e^{\phi}$ was introduced by Gromov [10] and it is a natural 
generalization of the mean curvature $H$ of a surface. A surface with $H_{\phi}=0$ is called a weighted minimal surface or a $\phi$-minimal surface in $\mathbb{R}^{3}$.

Another curvature for a surface in the Euclidean 3-space is the Gaussian curvature. In [11], authors introduced a generalized Gaussian curvature of a surface in a manifold with density $e^{\phi}$ and it is defined by

$$
G_{\phi}=G-\Delta \phi,
$$

where $G$ is the Riemannain Gaussian curvature of a surface and $\Delta$ is the Laplacian operator, which is called the weighted Gaussian curvature or the $\phi$-Gaussian curvature of a surface. Also, they obtained a generalization of the Gauss-Bonnet formula for a smooth disc in a smooth surface with density $e^{\phi}$.

For more details about manifolds with density and some relative topics, we refer readers to [12-17], etc. In particular, Hieu and Hoang [13] studied ruled surfaces and translation surfaces in $\mathbb{R}^{3}$ with density $e^{z}$ and they classified the weighted minimal ruled surfaces and translation surfaces. Lopez [15] considered a linear density $e^{a x+b y+c z}, a, b, c \in \mathbb{R}$, and he classified the weighted minimal translation surfaces and cyclic surfaces in a Euclidean 3-space $\mathbb{R}^{3}$. Also, Belarbi and Belkhelfa [18] investigated the properties of the weighted minimal graphs in $\mathbb{R}^{3}$ with a linear density.

In this article, we focus on a class of helicoidal surfaces in the Euclidean 3-space $\mathbb{R}^{3}$ with density $e^{\phi}$, where $\phi(p)=-x^{2}-y^{2}, p=(x, y, z) \in \mathbb{R}^{3}$. In particular, we construct all helicoidal surfaces in the space, in terms of the weighted Gaussian curvature and mean curvature, as smooth functions.

\section{Preliminaries}

We consider a regular plane curve $\gamma(u)=(g(u), 0, f(u))$ with $g(u)>0$ in the $x z$-plane which is defined on a open interval $I \subset \mathbb{R}$. A surface $M$ in the Euclidean 3-space $\mathbb{R}^{3}$ defined by

$$
X(u, v)=\left(\begin{array}{ccc}
\cos v & -\sin v & 0 \\
\sin v & \cos v & 0 \\
0 & 0 & 1
\end{array}\right)\left(\begin{array}{c}
g(u) \\
0 \\
f(u)
\end{array}\right)+h\left(\begin{array}{l}
0 \\
0 \\
v
\end{array}\right),
$$

where $h$ is a constant, is said to be the helicoidal surface with axis $O z$, a pitch $h$ and the profile curve $\gamma$. That is, $M$ can be parametrized by

$$
X(u, v)=(g(u) \cos v, g(u) \sin v, f(u)+h v) .
$$

We assume, without loss of generality, $\gamma(u)=(u, 0, f(u))$ is the profile curve in the $x z$-plane defined on any open interval $I$ of positive real numbers. Then, the helicoidal surface $M$ in $\mathbb{R}^{3}$ is given by

$$
X(u, v)=(u \cos v, u \sin v, f(u)+h v),
$$

where $f$ is a smooth function defined on $I$.

By a direct computation, the Gaussian curvature $G$ and the mean curvature $H$ of the surface are given by

$$
\begin{aligned}
& G=\frac{1}{D^{2}}\left[u^{3} f^{\prime}(u) f^{\prime \prime}(u)-h^{2}\right], \\
& H=\frac{1}{2 D^{\frac{3}{2}}}\left[\left(u^{2}+h^{2}\right) u f^{\prime \prime}(u)+u^{2} f^{\prime 3}(u)+\left(u^{2}+2 h^{2}\right) f^{\prime}(u)\right],
\end{aligned}
$$

where $D=\left(1+f^{\prime 2}(u)\right) u^{2}+h^{2}>0$. On the other hand, the unit normal vector $\mathbf{N}$ of the surface is

$$
\mathbf{N}=\frac{1}{\sqrt{D}}\left(h \sin v-u f^{\prime}(u) \cos v,-u f^{\prime}(u) \sin v-h \cos v, u\right) .
$$


Suppose that $M$ is the surface in $\mathbb{R}^{3}$ with density $e^{\phi}$, where $\phi=-x^{2}-y^{2}$. Then, in this case, the weighted mean curvature $H_{\phi}$ and the weighted Gaussian curvature $G_{\phi}$ can be expressed as

$$
H_{\phi}=\frac{1}{2 D^{\frac{3}{2}}}\left[\left(u^{2}+h^{2}\right) u f^{\prime \prime}(u)+\left(u^{2}-2 u^{4}\right) f^{\prime 3}(u)+\left(u^{2}+2 h^{2}-2 u^{4}-2 h^{2} u^{2}\right) f^{\prime}(u)\right]
$$

and

$$
G_{\phi}=\frac{1}{D^{2}}\left(u^{3} f^{\prime}(u) f^{\prime \prime}(u)-h^{2}\right)-4
$$

respectively.

\section{Main Theorems and Examples}

In this section, we construct helicoidal surfaces with prescribed weighted Gaussian curvature and weighted mean curvature in the Euclidean 3 -space $\mathbb{R}^{3}$ with density $e^{-x^{2}-y^{2}}$, where $(x, y, z) \in \mathbb{R}^{3}$.

\subsection{The Solution of Equation (5)}

Equation (5) is a second-order nonlinear ordinary differential equation. To solve it, we put

$$
A=\frac{f^{\prime}(u)}{\sqrt{D}} .
$$

Then, Equation (5) can be expressed in the form:

$$
H_{\phi}=u A^{\prime}+\left(2-2 u^{2}\right) A
$$

equivalently,

$$
A^{\prime}+\left(\frac{2}{u}-2 u\right) A=\frac{1}{u} H_{\phi} .
$$

It is a first-order linear ordinary differential equation with respect to $A$ and its general solution is given by

$$
A=\frac{e^{u^{2}}}{u^{2}}\left(\int u e^{-u^{2}} H_{\phi} d u+c_{1}\right),
$$

where $c_{1} \in \mathbb{R}$. On the other hand, Equations (7) and (9) imply

$$
\left[u^{2}-e^{2 u^{2}}\left(\int u e^{-u^{2}} H_{\phi} d u+c_{1}\right)^{2}\right] f^{\prime 2}(u)=\frac{u^{2}+h^{2}}{u^{2}} e^{2 u^{2}}\left(\int u e^{-u^{2}} H_{\phi} d u+c_{1}\right)^{2} .
$$

Since

$$
u^{2}-e^{2 u^{2}}\left(\int u e^{-u^{2}} H_{\phi} d u+c_{1}\right)^{2}=\frac{1}{D}\left(u^{4}+u^{2} h^{2}\right)>0,
$$

thus the general solution of Equation (10) becomes

$$
f(u)= \pm \int \frac{e^{u^{2}} \sqrt{u^{2}+h^{2}}\left(\int u e^{-u^{2}} H_{\phi} d u+c_{1}\right)}{u\left(u^{2}-e^{2 u^{2}}\left(\int u e^{-u^{2}} H_{\phi} d u+c_{1}\right)^{2}\right)^{\frac{1}{2}}} d u+c_{2}
$$

where $c_{2}$ is constant. 
Conversely, let $h$ be a given non-zero real constant and $H_{\phi}(u)$ be a real-valued smooth function defined on an open interval $I \subset(0,+\infty)$. Then, for any $u_{0} \in I$, there exist an open subinterval $I^{\prime}$ of $u_{0}$ $\left(I^{\prime} \subset I\right)$ and an open interval $J$ of $\mathbb{R}$ containing

$$
c_{1}^{\prime}=-\left(\int u e^{-u^{2}} H_{\phi} d u\right)\left(u_{0}\right)
$$

such that the function

$$
F\left(u, c_{1}\right)=u^{2}-e^{2 u^{2}}\left(\int u e^{-u^{2}} H_{\phi} d u+c_{1}\right)^{2}>0
$$

for any $\left(u, c_{1}\right) \in I^{\prime} \times J$. In fact, because $F\left(u_{0}, c_{1}^{\prime}\right)=u_{0}^{2}>0$, by the continuity of $F$, it is positive in a subset of $I^{\prime} \times J \subset \mathbb{R}^{2}$. Therefore, for any $\left(u, c_{1}\right) \in I^{\prime} \times J, c_{2} \in \mathbb{R}, h \in \mathbb{R}$ and any given smooth function $H_{\phi}$, we can define the two-parameter family of curves

$$
\gamma\left(u, H_{\phi}, h, c_{1}, c_{2}\right)=\left(u, 0, \pm \int \frac{e^{u^{2}} \sqrt{u^{2}+h^{2}}\left(\int u e^{-u^{2}} H_{\phi} d u+c_{1}\right)}{u\left(u^{2}-e^{2 u^{2}}\left(\int u e^{-u^{2}} H_{\phi} d u+c_{1}\right)^{2}\right)^{\frac{1}{2}}} d u+c_{2}\right) .
$$

Applying the one-parameter subgroup $\Phi_{t}^{h}$ on these curves, we can obtain a two-parameter family of helicoidal surfaces with the weighted mean curvature $H_{\phi}$.

Theorem 1. Let $\gamma(u)=(u, 0, f(u))$ be a profile curve of the helicoidal surface Equation (4) in the Euclidean 3 -space with density $e^{-x^{2}-y^{2}}$ of which the weighted mean curvature at the point $(u, 0, f(u))$ is given by $H_{\phi}(u)$. Then, for some constants $c_{1}, c_{2}$ and $h$, there exists the two-parameter family of helicoidal surfaces generated by plane curves

$$
\gamma\left(u, H_{\phi}(u), h, c_{1}, c_{2}\right)=\left(u, 0, \pm \int \frac{e^{u^{2}} \sqrt{u^{2}+h^{2}}\left(\int u e^{-u^{2}} H_{\phi} d u+c_{1}\right)}{u\left(u^{2}-e^{2 u^{2}}\left(\int u e^{-u^{2}} H_{\phi} d u+c_{1}\right)^{2}\right)^{\frac{1}{2}}} d u+c_{2}\right)
$$

Conversely, let $H_{\phi}(u)$ be a smooth function. Then, we can construct the two-parameter family of curves $\gamma\left(u, H_{\phi}(u), c_{1}, c_{2}\right)$ and so it is the two-parameter family of helicoidal surfaces with the weighted mean curvature $H_{\phi}(u)$ and a pitch $h$.

Corollary 1. Let M be a weighted minimal helicoidal surface in the Euclidean 3-space with density $e^{-x^{2}-y^{2}}$. Then, $M$ is an open part of either a helicoid or a surface parameterized by

$$
X(u, v)=(u \cos v, u \sin v, f(u)+h v),
$$

where

$$
f(u)= \pm \int \frac{c_{1} e^{u^{2}} \sqrt{u^{2}+h^{2}}}{u \sqrt{u^{2}-c_{1}^{2} e^{2 u^{2}}}} d u+c_{2}
$$

for some constants $c_{1}$ and $c_{2}$.

Proof. If $f$ is a constant function, it is a trivial solution for $H_{\phi}=0$. It follows that a helicoidal surface is a helicoid. Otherwise, we can easily compute Equation (11), in such case $f$ is given by (12).

Example 1. We consider a helicoidal surface with the constant weighted mean curvature

$$
H_{\phi}(u)=-2
$$


and the pitch $h=1$ in the Euclidean 3-space with density $e^{-x^{2}-y^{2}}$. Then, by Equation (11), we can calculate the profile curve $\gamma(u)$; from this, the parametrization of the surface is given by (see Figure 1)

$$
X(u, v)=\left(u \cos v, u \sin v, \frac{1}{2} \ln \left(2 u^{2}+2 \sqrt{u^{4}-1}\right)-\frac{1}{2} \tan ^{-1}\left(\frac{1}{\sqrt{u^{4}-1}}\right)+v\right) .
$$

Example 2. Consider a helicoidal surface with the weighted mean curvature

$$
H_{\phi}(u)=\frac{1}{\sqrt{2} u}\left(1-2 u^{2}\right)
$$

and the pitch $h=1$ in the Euclidean 3-space with density $e^{-x^{2}-y^{2}}$. By a direct computation with the help of Equation (11), we can compute the profile curve $\gamma(u)$, which implies that the parametrization of the surface is expressed in the form (see Figure 2):

$$
X(u, v)=\left(u \cos v, u \sin v, \sqrt{u^{2}+1}-\tan ^{-1}\left(\frac{1}{\sqrt{u^{2}+1}}\right)+v\right) .
$$

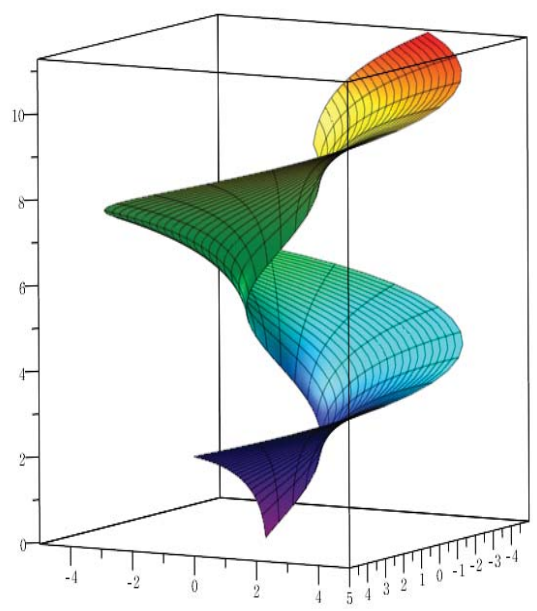

Figure 1. A helicoidal surface with $H_{\phi}(u)=-2$.

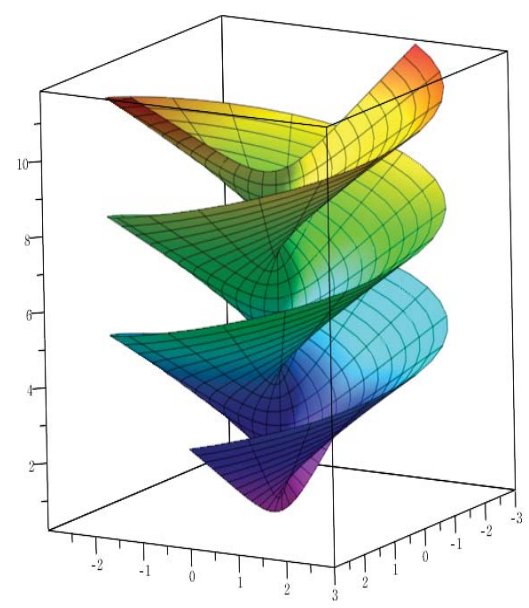

Figure 2. A helicoidal surface with $H_{\phi}(u)=\frac{1}{\sqrt{2} u}\left(1-2 u^{2}\right)$. 


\subsection{The Solution of Equation (6)}

To solve the second-order nonlinear ordinary differential Equation (6), we put

$$
B=\frac{u^{2} f^{\prime 2}(u)+h^{2}}{D} .
$$

Then, the weighted Gaussian curvature $G_{\phi}$ can be rewritten as:

$$
G_{\phi}=\frac{1}{2 u} B^{\prime}-4
$$

that is,

$$
B^{\prime}=2 u G_{\phi}+8 u
$$

The solution of the last equation is

$$
B=4 u^{2}+\int 2 u G_{\phi} d u+c_{1}
$$

for some constant $c_{1}$. Combining Equations (13) and (15), one gets

$$
u^{2}\left(1-4 u^{2}-\int 2 u G_{\phi} d u-c_{1}\right) f^{\prime 2}(u)=\left(u^{2}+h^{2}\right)\left(4 u^{2}+\int 2 u G_{\phi} d u+c_{1}\right)-h^{2} .
$$

Since

$$
1-4 u^{2}-\int 2 u G_{\phi} d u-c_{1}=\frac{u^{2}}{D}>0,
$$

the general solution of Equation (16) is given by

$$
f(u)= \pm \int \frac{1}{u}\left[\frac{\left(u^{2}+h^{2}\right)\left(4 u^{2}+\int 2 u G_{\phi} d u+c_{1}\right)-h^{2}}{1-4 u^{2}-\int 2 u G_{\phi} d u-c_{1}}\right]^{\frac{1}{2}} d u+c_{2}
$$

where $c_{2} \in \mathbb{R}$.

Conversely, let $h$ be a given real number and $G_{\phi}$ be a smooth function defined on an open interval $I \subset(0,+\infty)$. Let

$$
F\left(u, c_{1}\right)=1-4 u^{2}-\int 2 u G_{\phi} d u-c_{1}
$$

be a function defined on $I \times \mathbb{R} \subset \mathbb{R}^{2}$. For any $u_{0} \in I$, denote

$$
c_{1}^{\prime}=-\left(4 u^{2}+\int 2 u G_{\phi} d u\right)\left(u_{0}\right) .
$$

Thus, we can find an open subinterval $I^{\prime} \subset I$ containing $u_{0}$ and an open interval $J$ of $\mathbb{R}$ containing $c_{1}^{\prime}$ such that the function $F\left(u, c_{1}\right)$ is positive for any $\left(u, c_{1}\right) \in I^{\prime} \times J$. In fact, because $F\left(u_{0}, c_{1}^{\prime}\right)=1$, by the continuity of $F$, it is positive in a subset of $I^{\prime} \times J \subset \mathbb{R}^{2}$. Theeqrefore, for any $\left(u, c_{1}\right) \in I^{\prime} \times J$, $h \in \mathbb{R}, c_{2} \in \mathbb{R}$ and given the smooth function $G_{\phi}$, we can define the two-parameter family of curves

$$
\gamma\left(u, G_{\phi}, h, c_{1}, c_{2}\right)=\left(u, 0, \pm \int \frac{1}{u}\left[\frac{\left(u^{2}+h^{2}\right)\left(4 u^{2}+\int 2 u G_{\phi} d u+c_{1}\right)-h^{2}}{1-4 u^{2}-\int 2 u G_{\phi} d u-c_{1}}\right]^{\frac{1}{2}} d u+c_{2}\right) .
$$

Consequently, we get a two-parameter family of helicoidal surfaces with the weighted Gaussian curvature $G_{\phi}(u), u \in I^{\prime}$ and we have the following theorem. 
Theorem 2. Let $\gamma(u)=(u, 0, f(u))$ be a profile curve of the helicoidal surface (4) in the Euclidean 3-space with density $e^{-x^{2}-y^{2}}$ of which the weighted Gaussian curvature at the point $(u, 0, f(u))$ is given by $G_{\phi}(u)$. Then, for some constants $c_{1}, c_{2}$ and $h$, there exists the two-parameter family of the helicoidal surface generated by plane curves

$$
\gamma\left(u, G_{\phi}, h, c_{1}, c_{2}\right)=\left(u, 0, \pm \int \frac{1}{u}\left[\frac{\left(u^{2}+h^{2}\right)\left(4 u^{2}+\int 2 u G_{\phi} d u+c_{1}\right)-h^{2}}{1-4 u^{2}-\int 2 u G_{\phi} d u-c_{1}}\right]^{\frac{1}{2}} d u+c_{2}\right)
$$

Conversely, let $G_{\phi}(u)$ be a smooth function. Then, for any $u_{0} \in I$, we can construct the two-parameter family of curves $\gamma\left(u, G_{\phi}(u), h, c_{1}, c_{2}\right), u \in I^{\prime} \subset I$ and so it is the two-parameter family of helicoidal surfaces with the weighted Gaussian curvature $G_{\phi}(u), u \in I^{\prime}$.

Example 3. We consider a helicoidal surface in the Euclidean 3-space with density $e^{-x^{2}-y^{2}}$ with a negative weighted Gaussian curvature

$$
G_{\phi}(u)=-\frac{1}{\left(2 u^{2}+1\right)^{2}}-4 .
$$

In such a case, an integration of Equation (17) implies $f(u)=u$ for $h=1, c_{1}=0$ and $c_{2}=0$. It follows that the helicoidal surface is parametrized by (see Figure 3)

$$
X(u, v)=(u \cos v, u \sin v, u+v) .
$$

Example 4. Consider a helicoidal surface in the Euclidean 3-space with density $e^{-x^{2}-y^{2}}$ with a weighted Gaussian curvature

$$
G_{\phi}(u)=\frac{2 u^{2}-1}{\left(u^{4}-u^{2}-1\right)^{2}}-4 .
$$

Then, from Equation (17), we have $f(u)=\sin ^{-1} u$ for $h=1, c_{1}=0$ and $c_{2}=0$ and, in this case, a parametrization of the helicoidal surface is given by (see Figure 4)

$$
X(u, v)=\left(u \cos v, u \sin v, \sin ^{-1} u+v\right) .
$$

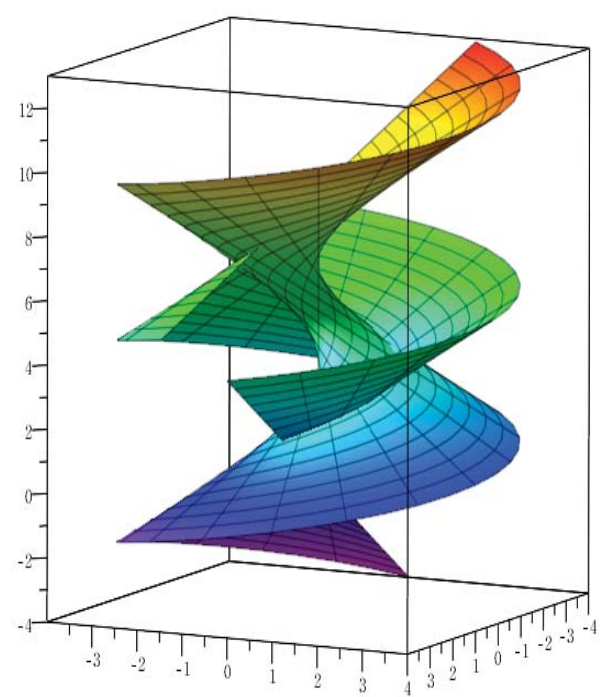

Figure 3. A helicoidal surface with $G_{\phi}(u)=-\frac{1}{\left(2 u^{2}+1\right)^{2}}-4$. 


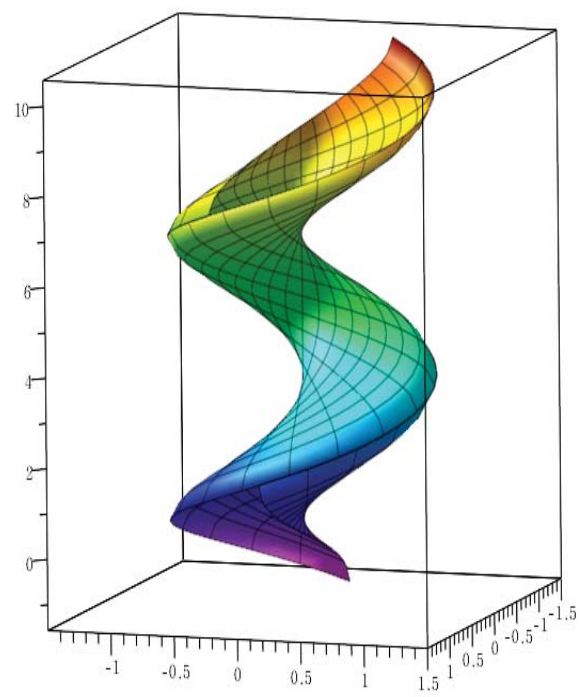

Figure 4. A helicoidal surface with $G_{\phi}(u)=\frac{2 u^{2}-1}{\left(u^{4}-u^{2}-1\right)^{2}}-4$.

Acknowledgments: We would like to thank the referee for the careful review and the valuable comments, which really improved the paper. The first author was supported by the Basic Science Research Program through the National Research Foundation of Korea (NRF) funded by the Ministry of Education (2015R1D1A1A01060046). The fourth author was supported by Basic Science Research Program through the National Research Foundation of Korea(NRF) funded by the Ministry of Education(2017R1D1A1B03033978).

Author Contributions: Dae Won Yoon and Jae Won Lee gave the idea for the prescribed curvature problem in a manifold with density. Dong-Soo Kim and Young Ho Kim checked and polished the draft.

Conflicts of Interest: The authors declare no conflict of interest.

\section{References}

1. Kenmotsu, K. Surfaces of revolution with prescribed mean curvature. Tôhoku Math. J. 1980, 32, 147-153.

2. Delaunay, G. Sur la surface de revolution dont la courbure moyenne est constante. J. Math. Pures Appl. 1841, 6, 309-320.

3. Baikoussis, C.; Koufogiorgos, T. Helicoidal surfaces with prescribed mean or Gaussian curvature. J. Geom. 1998, 63, 25-29.

4. Beneki, C.C.; Kaimakamis, G.; Papantonios, B.J. Helicoidal surfaces in three-dimensional Minkowski space. J. Math. Anal. Appl. 2002, 275, 586-614.

5. Ji, F.H.; Hou, Z.H. A kind of helicoidal surfaces in 3-dimensional Minkowski space. J. Math. Anal. Appl. 2005, 304, 623-643.

6. Ji, F.; Hou, Z.H. Helicoidal surfaces under the cubic screw motion in Minkowski 3-space. J. Math. Anal. Appl. 2006, 318, 634-647.

7. Hou, Z.H.; Ji, F.H. Helicoidal surfaces with $H^{2}=K$ in Minkowski 3-space. J. Math. Anal. Appl. 2007, 325, 101-113.

8. Yoon, D.W.; Kim, D.-S.; Kim, Y. H.; Lee, J.W. Helicoidal surfaces with prescribed curvatures in $\mathrm{Nil}_{3}$. Int. J. Math. 2013, 24, 1350107 .

9. Morgan, F. Manifolds with density and Perelman's proof of the Poincare Conjecture. Am. Math. Mon. 2009, 116, 134-142.

10. Gromov, M. Isoperimetry of waists and concentration of maps. Geom. Funct. Anal. 2003, 13, 178-215.

11. Corwin, I.; Hoffman, H.; Hurder, S.; Ššum, V.; Xu, Y. Differential geometry of manifolds with density. Rose-Hulman Undergrad. Math. J. 2006, 7, 1-15.

12. Carroll, C.; Jacob, A.; Quinn, C.; Walters, R. The isoperimetric problem on planes with density. Bull. Aust. Math. Soc. 2008, 78, 177-197.

13. Hieu, D.T.; Hoang, N.M. Ruled minimal surfaces in $\mathbb{R}^{3}$ with density $e^{z}$. Pac. J. Math. 2009, 243, $277-285$. 
14. Hieu, D.T.; Nam, T.L. The classification of constant weighted curvature curves in the plane with a log-linear density. Commun. Pure Appl. Anal. 2014, 13, 1641-1652.

15. López, R. Minimal surface in Euclidean Space with a Log-Linear Density. Available online: https:/ / arxiv.org/abs/1410.2517 (accessed on 20 July 2017).

16. Rosales, C.; Canete, A.; Bayle, V.; Morgan, F. On the isoperimetric problem in Euclidean space with density. Calc. Var. PDE 2008, 31, 27-46.

17. Yoon, D.W.; Lee, J.W.; Lee, C.W. $\phi$-minimal rotational surfaces in pseudo-Galilean space with density. Ann. Pol. Math. 2017, submitted.

18. Belarbi, L.; Belkhelfa, M. Surfaces in $\mathbb{R}^{3}$ with density. I-Manag. J. Math. 2012, 1, 34-48.

(C) 2017 by the authors. Licensee MDPI, Basel, Switzerland. This article is an open access article distributed under the terms and conditions of the Creative Commons Attribution (CC BY) license (http://creativecommons.org/licenses/by/4.0/). 